\title{
Gaisböck syndrome
}

INSERM

\section{Source}

INSERM. (1999). Orphanet: an online rare disease and orphan drug data base. Gaisböck syndrome. ORPHA:90041

Gaisbock syndrome is characterised by secondary polycythemia. 